PROCEEDINGS OF THE

AMERICAN MATHEMATICAL SOCIETY

Volume 132, Number 4, Pages 1153-1162

S 0002-9939(03)07189-2

Article electronically published on August 28, 2003

\title{
MANSFIELD'S IMPRIMITIVITY THEOREM FOR ARBITRARY CLOSED SUBGROUPS
}

\author{
ASTRID AN HUEF AND IAIN RAEBURN
}

(Communicated by David R. Larson)

\begin{abstract}
Let $\delta$ be a nondegenerate coaction of $G$ on a $C^{*}$-algebra $B$, and let $H$ be a closed subgroup of $G$. The dual action $\hat{\delta}: H \rightarrow \operatorname{Aut}\left(B \times{ }_{\delta} G\right)$ is proper and saturated in the sense of Rieffel, and the generalised fixed-point algebra is the crossed product of $B$ by the homogeneous space $G / H$. The resulting Morita equivalence is a version of Mansfield's imprimitivity theorem which requires neither amenability nor normality of $H$.
\end{abstract}

\section{INTRODUCTION}

Nonabelian duality tells us how to recover information about a $C^{*}$-dynamical system $(A, G, \alpha)$ from its crossed product $A \times{ }_{\alpha} G$. The dual systems consist of a coaction $\delta: B \rightarrow M\left(B \otimes C_{r}^{*}(G)\right)$ of a locally compact group $G$ on a $C^{*}$-algebra $B$; one recovers $(A, G, \alpha)$ from $A \times{ }_{\alpha} G$ by taking the crossed product $\left(A \times_{\alpha} G\right) \times{ }_{\hat{\alpha}} G$ by the dual coaction $\hat{\alpha}$, and invoking the duality theorem of Imai and Takai. The theory of crossed products by coactions runs parallel to the theory of ordinary crossed products by actions, and there are now analogues for coactions of many of the important theorems about ordinary crossed products. In particular, Mansfield has proved an imprimitivity theorem for crossed products by coactions analogous to that of Rieffel and Green for ordinary crossed products [8].

Let $\delta: B \rightarrow M\left(B \otimes C_{r}^{*}(G)\right)$ be a coaction of a locally compact group $G$, and let $N$ be a closed subgroup of $G$ which is both normal and amenable. The coaction $\delta$ restricts to a coaction $\delta \mid$ of $G / N$, and Mansfield's imprimitivity theorem says that $B \times_{\delta \mid}(G / N)$ is Morita equivalent to the crossed product $\left(B \times{ }_{\delta} G\right) \times_{\hat{\delta}} N$ by the restriction of the dual action. This theorem is widely regarded as the deepest result in the subject, and Mansfield's analysis is subtle and complicated.

The amenability hypothesis in Mansfield's theorem ensures that the restriction $\delta \mid$ is well defined. If one considers instead full coactions $\epsilon: B \rightarrow M\left(B \otimes C^{*}(G)\right)$, then the restriction $\epsilon \mid$ makes sense for nonamenable $N$, and Kaliszewski and Quigg showed that Mansfield's theorem extends to many, but not all, full coactions [6]. Recently there have been indications that one might be able to extend Mansfield's theorem to nonnormal subgroups [2], [5], and indeed such an extension has been proved for discrete groups [3].

Received by the editors June 30, 2002 and, in revised form, December 18, 2002.

2000 Mathematics Subject Classification. Primary 46L05; Secondary 46L08, 46L55.

This research was supported by grants from the Australian Research Council, the University of New South Wales and the University of Newcastle. 
Here we prove a version of Mansfield's imprimitivity theorem which requires neither amenability nor normality of the subgroup. Our result contains interesting new information, even for amenable nonnormal subgroups (Corollary 3.5) and for normal nonamenable subgroups (Corollary 4.3). We use several powerful tools: Rieffel's theory of proper actions [16], [17, the dense subalgebras constructed by Mansfield in [8, §3], an averaging technique developed by Quigg [12], 14], and the crossed products by homogeneous spaces introduced in 2 .

We begin with a short section in which we establish conventions and notation, and then prove our main Theorem 3.1 in Section 3 . Our proof is quite different from Mansfield's, and is substantially shorter because we are taking advantage of established technology. We prove that the dual action $\hat{\delta}$ of a closed subgroup $H$ on $B \times{ }_{\delta} G$ is proper and saturated with respect to Mansfield's subalgebra $\mathcal{D}$, and then identify the associated fixed-point algebra with the crossed product of $B$ by the homogeneous space $G / H$. In Section 4 we look at what happens when $H=N$ is normal. We use results of Quigg [13] to define the restriction of a reduced coaction $\delta$ of $G$ to a reduced coaction $\delta \mid$ of $G / N$, identify the crossed product by $\delta \mid$ with the crossed product by the homogeneous space (Proposition 4.2), and deduce a very satisfactory version of Mansfield's theorem for $B \times_{\delta \mid}(G / N)$. We then reconcile our results with those of Kaliszewski and Quigg for full coactions.

\section{Preliminaries}

Let $G$ be a locally compact group and $\lambda^{G}=\lambda$ the left-regular representation of $G$ on $L^{2}(G)$. We view the reduced group $C^{*}$-algebra $C_{r}^{*}(G)$ as the subalgebra $\lambda\left(C^{*}(G)\right)$ of $B\left(L^{2}(G)\right)$. We use minimal tensor products throughout. We routinely extend nondegenerate homomorphisms to multiplier algebras without changing notation.

In Section 3 we use the conventions of [7]. Thus $\delta_{G}$ denotes the reduced comultiplication $\delta_{G}: C_{r}^{*}(G) \rightarrow M\left(C_{r}^{*}(G) \otimes C_{r}^{*}(G)\right)$ characterised by $\delta_{G}\left(\lambda_{s}\right)=\lambda_{s} \otimes \lambda_{s}$, and a reduced coaction $\delta$ of $G$ on a $C^{*}$-algebra $B$ is an injective nondegenerate homomorphism $\delta: B \rightarrow M\left(B \otimes C_{r}^{*}(G)\right)$ such that $(\delta \otimes \iota) \circ \delta=\left(\iota \otimes \delta_{G}\right) \circ \delta$ and $\delta(b)(1 \otimes z) \in B \otimes C_{r}^{*}(G)$ for all $b \in B$ and $z \in C_{r}^{*}(G)$. We write $A_{c}(G)$ for the set of functions in the Fourier algebra $A(G)$ with compact support. For $u \in A(G)$ and $b \in B$ we write $\delta_{u}(b):=(\iota \otimes u)(\delta(b))$; the coaction is nondegenerate if $\overline{\delta_{A(G)}(B)}=B$. Our main Theorem 3.1 like Mansfield's, is about reduced coactions. In Section 4 we need to use full coactions, and our conventions there are also the standard ones.

Let $\pi$ be a faithful nondegenerate representation of $B$ on $\mathcal{H}_{\pi}$ and $M$ the representation of $C_{0}(G)$ by multiplication operators on $L^{2}(G)$. The closed span

$$
B \times{ }_{\delta} G:=\overline{\operatorname{sp}}\left\{((\pi \otimes \iota) \circ \delta(b))(1 \otimes M(f)): b \in B, f \in C_{0}(G)\right\} .
$$

is a $C^{*}$-subalgebra of $B\left(\mathcal{H}_{\pi} \otimes L^{2}(G)\right)$, which is up to isomorphism independent of the representation $\pi$, and which is called the crossed product of $B$ by $\delta$. Conjugation by the right-regular representation gives a dual action $\hat{\delta}$ of $G$ on $B \times{ }_{\delta} G$, which is characterised by

$$
\hat{\delta}_{s}(((\pi \otimes \iota)(\delta(b)))(1 \otimes M(f)))=((\pi \otimes \iota)(\delta(b)))\left(1 \otimes M\left(\mathrm{rt}_{s}(f)\right)\right)
$$

where $\operatorname{rt}_{s}(f)(t)=f(t s)$.

Let $H$ be a closed subgroup of $G$. As in [2, Definition 2.1], the reduced crossed product $B \times_{\delta, r}(G / H)$ of $B$ by the "coaction" of the homogeneous space $G / H$ is 
the $C^{*}$-subalgebra of $B\left(\mathcal{H}_{\pi} \otimes L^{2}(G)\right)$ generated by the operators

$$
\left\{((\pi \otimes \iota) \circ \delta(b))(1 \otimes M \mid(f)): b \in B, f \in C_{0}(G / H)\right\},
$$

where $M \mid$ denotes the extension of $M$ to $C_{0}(G / H) \subset M\left(C_{0}(G)\right)$. (The reasons for calling this the reduced crossed product are discussed in [2, §2].) It follows from [8. Lemma 11] that

$$
B \times_{\delta, r}(G / H)=\overline{\operatorname{sp}}\left\{((\pi \otimes \iota) \circ \delta(b))(1 \otimes M \mid(f)): b \in B, f \in C_{0}(G / H)\right\} .
$$

Suppose that $E$ is a compact subset of $G$ and $u \in A_{c}(G)$. Denote by $C_{E}(G)$ the set of functions in $C_{c}(G)$ with support in $E$. Following Mansfield, we say that an operator on $\mathcal{H}_{\pi} \otimes L^{2}(G)$ is $(u, E)$ if it is the norm limit of a sequence $\left\{x_{i}\right\}$ of the form

$$
x_{i}=\sum_{j=1}^{n_{i}}(\pi \otimes \iota)\left(\delta\left(\delta_{u}\left(b_{i j}\right)\right)\right)\left(1 \otimes M\left(f_{i j}\right)\right) \text { where } f_{i j} \in C_{E}(G) .
$$

Then $\mathcal{D}$ denotes the set of operators that are $(u, E)$ for some $u \in A_{c}(G)$ and compact subset $E$ of $G[8, \S 3]$. The point of the definition is that one can pull $\pi \otimes \iota\left(\delta\left(\delta_{u}(b)\right)\right)$ past $(1 \otimes M(f))$ and remain within $\mathcal{D}[8$, Lemma 9] so that $\mathcal{D}$ is a *-subalgebra of $B \times{ }_{\delta} G[\underline{8}$, Lemma 11]; if $\delta$ is nondegenerate, then $\mathcal{D}$ is dense in $B \times{ }_{\delta} G$.

\section{THE MAIN THEOREM}

Theorem 3.1. Suppose that $\delta$ is a nondegenerate reduced coaction of a locally compact group $G$ on a $C^{*}$-algebra $B$ and that $H$ is a closed subgroup of $G$. Then the restriction of the dual action $\hat{\delta}: G \rightarrow \operatorname{Aut}\left(B \times_{\delta} G\right)$ to $H$ is proper and saturated with respect to Mansfield's subalgebra $\mathcal{D}$, and the generalised fixed-point algebra is the crossed product $B \times_{\delta, r}(G / H)$ of $B$ by the homogeneous space $G / H$. Thus, by [16. Corollary 1.7], D completes to give a $\left(\left(B \times{ }_{\delta} G\right) \times_{\hat{\delta}, r} H\right)-\left(B \times{ }_{\delta, r}(G / H)\right)$ imprimitivity bimodule.

When the subgroup $H$ is normal and amenable, all of this was proved by Mansfield in 8], though he proved directly that $\mathcal{D}$ completes to give an imprimitivity bimodule, and deduced from his arguments that the dual action of $H$ is proper. For general $H$, it is known that $\left(B \times_{\delta} G\right) \times_{\hat{\delta}, r} H$ and $B \times_{\delta, r}(G / H)$ are Morita equivalent 2, $\S 5]$; however, the argument in [2] is indirect, and [2, Theorem 5.2] generalises the weaker version of Mansfield's theorem proved in 9] rather than Mansfield's theorem itself. Theorem 3.1 generalises Mansfield's theorem, and gives a concrete bimodule which can be used to induce representations.

The idea of approaching Mansfield's theorem through properness comes from [5] and [1], where Theorem 3.1] was proved for discrete groups ([1, Theorem 5.1]). We know from recent work of Rieffel [17, Theorem 5.7] that $\hat{\delta}$ is proper and saturated with respect to the $*$-subalgebra

$$
A_{0}:=\left(1 \otimes M\left(C_{c}(G)\right)\right)\left(B \times{ }_{\delta} G\right)\left(1 \otimes M\left(C_{c}(G)\right)\right)
$$

of $B \times{ }_{\delta} G$ (see [5] Remark 4.5]). It is not obvious to us, however, how to directly identify the generalised fixed-point algebra associated to $A_{0}$. Our main observation is that if we use $\mathcal{D}$ instead of $A_{0}$, then we can use Mansfield's results to help identify the generalised fixed-point algebra with $B \times_{\delta, r}(G / H)$. The catch is that we cannot 
then use the results of [17], and have to verify directly that $\hat{\delta}$ is proper with respect to $\mathcal{D}$.

Our first lemma says that $\mathcal{D}$ is smaller than Rieffel's $A_{0}$.

Lemma 3.2. Suppose $u \in A_{c}(G)$ and $E$ is a compact subset of $G$. Then there exist $f, g \in C_{c}(G)$ such that $x=(1 \otimes M(f)) x(1 \otimes M(g))$ for every $x \in \mathcal{D}$ which is $(u, E)$; in particular, $\mathcal{D}=\left(1 \otimes M\left(C_{c}(G)\right)\right) \mathcal{D}\left(1 \otimes M\left(C_{c}(G)\right)\right)$.

Proof. Choose $g \in C_{c}(G)$ with $g=1$ on $E$. Then a glance at (2.1) shows that $x=x(1 \otimes M(g))$ whenever $x$ is $(u, E)$. Let $\tilde{u}(t)=\overline{u\left(t^{-1}\right)}$. Then by [8, Lemma 11(ii)], there is a compact subset $F$ such that $x^{*}$ is $(\tilde{u}, F)$ whenever $x$ is $(u, E)$. Now choose $f \in C_{c}(G)$ such that $f=1$ on $F$, and we have $(1 \otimes M(f)) x=x$.

To establish that $\hat{\delta}$ is proper, we verify the conditions of [16 Definition 1.2]: for every $x, y \in \mathcal{D}$,

1. both $s \mapsto x \hat{\delta}_{s}\left(y^{*}\right)$ and $s \mapsto \Delta(s)^{-1 / 2} x \hat{\delta}_{s}\left(y^{*}\right)$ are integrable, and

2. there exists $\langle x, y\rangle \in M\left(B \times{ }_{\delta} G\right)$ such that for all $z \in \mathcal{D}$,

$$
z\langle x, y\rangle \in \mathcal{D} \text { and } z\langle x, y\rangle=\int_{H} z \hat{\delta}_{s}\left(x^{*} y\right) d s .
$$

Given Lemma 3.2, item 1 is easy: factor $x=x(1 \otimes M(f))$ and $y=y(1 \otimes M(g))$, so that

$$
x \hat{\delta}_{s}\left(y^{*}\right)=x\left(1 \otimes M\left(f \operatorname{rt}_{s}(\bar{g})\right)\right) \hat{\delta}_{s}\left(y^{*}\right),
$$

and note that $s \mapsto f \operatorname{rt}_{s}(\bar{g})$ has compact support.

So the crux in proving that $\hat{\delta}$ is proper is to find the multipliers $\langle x, y\rangle$. To do this, we use an averaging technique which was developed by Olesen and Pedersen [10], 11], applied to coactions by Quigg [12], [14, and shown to be relevant to properness by Rieffel [17. Let $\alpha$ be an action of a locally compact group $K$ on a $C^{*}$-algebra $A$. Denote by $\mathfrak{p}$ the set of multipliers $a \in M(A)^{+}$for which there exists $\Psi(a) \in M(A)^{+}$satisfying

$$
\omega(\Psi(a))=\int_{K} \omega\left(\alpha_{s}(a)\right) d s \text { for all } \omega \in A^{*}
$$

(where we have implicitly extended $\omega$ to a strictly continuous functional on $M(A)$ and $\alpha$ to a strictly continuous action on $M(A)$ ). The functionals $\omega \in A^{*}$ separate points of $M(A)$, so $\Psi(a)$ is uniquely determined by (3.3). By [12, Corollary 3.6], the linear span $\mathfrak{m}$ of $\mathfrak{p}$ is a $*$-subalgebra of $M(A)$ with $\mathfrak{m}^{+}=\mathfrak{p}$, and $\Psi$ extends uniquely to a positive linear map $\Psi: \mathfrak{m} \rightarrow M(A)$ such that $a \mapsto \Psi(f a g)$ is norm-continuous on $M(A)$ for fixed $f$ and $g$ in $\mathfrak{m}$.

We now describe the averaging map $\Psi$ for $(A, K, \alpha)=\left(B \times_{\delta} G, H, \hat{\delta}\right)$. For $f \in C_{c}(G)$, we define $\Phi(f) \in C_{c}(G / H)$ by

$$
\Phi(f)(t H)=\int_{H} f(t s) d s .
$$

Lemma 3.3. Rieffel's subalgebra $A_{0}$ of (3.1) is contained in $\mathfrak{m}$, and, in particular, $\mathcal{D} \subset \mathfrak{m}$. For $b \in B, u \in A_{c}(G)$ and $f \in C_{c}(G)$ we have

$$
\Psi\left((\pi \otimes \iota)\left(\delta\left(\delta_{u}(b)\right)\right)(1 \otimes M(f))\right)=(\pi \otimes \iota)\left(\delta\left(\delta_{u}(b)\right)\right)(1 \otimes M(\Phi(f))) .
$$


Proof. (These arguments are implicit in [12, §3] and [14, §1]; see also [17, §§4-5].) We first claim that $f \in \mathfrak{m}\left(C_{0}(G)\right.$, rt), with $\Psi(f)=\Phi(f)$. To see this, it suffices to take $f \in C_{c}(G)^{+}, \omega \in C_{c}(G)^{*+}$ and show that

$$
\omega(\Phi(f))=\int_{H} \omega\left(\mathrm{rt}_{s}(f)\right) d s .
$$

But the Riesz Representation Theorem says that $\omega$ is given by a finite Borel measure $\mu$, and then (3.5) reduces to Tonelli's Theorem.

Now [14, Proposition 1.4] implies that $1 \otimes M(f)$ belongs to $\mathfrak{m}\left(B \times{ }_{\delta} G, H, \hat{\delta}\right)$; in fact, it belongs to the left ideal $\mathfrak{n}$ of [12, Definition 3.4], and hence every element of the form $(1 \otimes M(f)) c(1 \otimes M(g))$ for $c \in B \times{ }_{\delta} G$ belongs to $\mathfrak{m}=\mathfrak{n}^{*} \mathfrak{n}$. Thus $A_{0} \subset \mathfrak{m}$.

To see (3.4), let $\kappa \in\left(B \times{ }_{\delta} G\right)^{*}$ and define a bounded functional $\omega$ on $C_{0}(G)$ by

$$
\omega(f):=\kappa\left(\pi \otimes \iota\left(\delta\left(\delta_{u}(b)\right)\right)(1 \otimes M(f))\right) .
$$

Equation (3.5) extends to arbitrary bounded functionals by linearity. So

$$
\begin{aligned}
\kappa\left(\pi \otimes \iota\left(\delta\left(\delta_{u}(b)\right)(1 \otimes M(\Phi(f)))\right)\right. & =\omega(\Phi(f))=\int_{H} \omega\left(\mathrm{rt}_{s}(f)\right) d s \\
& =\int_{H} \kappa\left(\pi \otimes \iota\left(\delta\left(\delta_{u}(b)\right)\right)\left(1 \otimes M\left(\mathrm{rt}_{s}(f)\right)\right)\right) d s \\
& =\int_{H} \kappa\left(\hat{\delta}_{s}\left(\pi \otimes \iota\left(\delta\left(\delta_{u}(b)\right)\right)\right)(1 \otimes M(f))\right) d s,
\end{aligned}
$$

and this implies (3.4).

To finish the proof of Theorem 3.1, we need the subalgebra $\mathcal{D}_{H}$ constructed in 8, $\S 3]$; notice that Mansfield was careful not to use amenability or normality in that section. Let $E$ be a compact subset of $G$ and $u \in A_{c}(G)$. An operator on $\mathcal{H}_{\pi} \otimes L^{2}(G)$ is $(u, E, H)$ if it is the norm limit of a sequence $\left\{x_{i}\right\}$ of the form

$$
x_{i}=\sum_{j=1}^{n_{i}}\left((\pi \otimes \iota)\left(\delta\left(\delta_{u}\left(b_{i j}\right)\right)\right)\right)\left(1 \otimes M \mid\left(\Phi\left(f_{i j}\right)\right)\right) \text { where } f_{i j} \in C_{E}(G) .
$$

Then $\mathcal{D}_{H}$ is the set of operators on $\mathcal{H}_{\pi} \otimes L^{2}(G)$ that are $(u, E, H)$ for some $u \in$ $A_{c}(G)$ and compact subset $E$ of $G$.

The end of the proof of Theorem 3.1. Given our earlier observations, to show that $\hat{\delta}$ is a proper action we need to define multipliers $\langle x, y\rangle$ of $\mathcal{D}$ satisfying (3.2). We set $\langle x, y\rangle:=\Psi\left(x^{*} y\right)$.

We first claim that $\langle x, y\rangle \in \mathcal{D}_{H}$; this gives $z\langle x, y\rangle \in \mathcal{D}$ for $z \in \mathcal{D}$ because $\mathcal{D}_{H}$ multiplies $\mathcal{D}[8$, Lemma $11(\mathrm{v})]$. Since $\mathcal{D}$ is a $*$-algebra we can write $x^{*} y$ as the norm limit of

$$
x_{i}=\sum_{j=1}^{n_{i}}\left((\pi \otimes \iota)\left(\delta\left(\delta_{u}\left(b_{i j}\right)\right)\right)\right)\left(1 \otimes M\left(f_{i j}\right)\right) \text { where each } x_{i} \text { is }(u, E) .
$$

By Lemma 3.2 there are $f, g \in C_{c}(G)$ such that $x_{i}=(1 \otimes M(f)) x_{i}(1 \otimes M(g))$ for all $i$, and then $x^{*} y=(1 \otimes M(f)) x^{*} y(1 \otimes M(g))$ as well. The point is that since $1 \otimes M(f)$ and $1 \otimes M(g)$ belong to $\mathfrak{m}$, the map $c \mapsto \Psi((1 \otimes M(f)) c(1 \otimes M(g)))$ is 
norm-continuous on $B \times{ }_{\delta} G$ by [12, Corollary 3.6]. Thus we have

$$
\begin{aligned}
\langle x, y\rangle=\Psi\left(x^{*} y\right) & =\Psi\left((1 \otimes M(f))\left(\lim _{i} x_{i}\right)(1 \otimes M(g))\right) \\
& =\lim _{i} \Psi\left((1 \otimes M(f))\left(x_{i}\right)(1 \otimes M(g))\right) \\
& =\lim _{i} \Psi\left(\sum_{j=1}^{n_{i}}\left((\pi \otimes \iota)\left(\delta\left(\delta_{u}\left(b_{i j}\right)\right)\right)\right)\left(1 \otimes M\left(f_{i j}\right)\right)\right),
\end{aligned}
$$

and it follows from Lemma 3.3 that

$$
\langle x, y\rangle=\lim _{i} \sum_{j=1}^{n_{i}}\left((\pi \otimes \iota)\left(\delta\left(\delta_{u}\left(b_{i j}\right)\right)\right)\right)\left(1 \otimes M\left(\Phi\left(f_{i j}\right)\right)\right) .
$$

Thus $\langle x, y\rangle$ is $(u, E, H)$, and belongs to $\mathcal{D}_{H}$, as claimed.

Next suppose $z \in \mathcal{D}$ and $\kappa \in\left(B \times{ }_{\delta} G\right)^{*}$, and define a bounded linear functional $\omega$ on $B \times_{\delta} G$ by $\omega(a)=\kappa(z a)$. Equation (3.3) gives

$$
\kappa(z\langle x, y\rangle)=\omega\left(\Psi\left(x^{*} y\right)\right)=\int_{H} \omega\left(\hat{\delta}_{s}\left(x^{*} y\right)\right) d s=\int_{H} \kappa\left(z \hat{\delta}_{s}\left(x^{*} y\right)\right) d s,
$$

which equals $\kappa\left(\int_{H} z \hat{\delta}_{s}\left(x^{*} y\right) d s\right)$ because the integrand is integrable and bounded linear maps pull through integrals. So $z\langle x, y\rangle=\int_{H} z \hat{\delta}_{s}\left(x^{*} y\right) d s$, and we have found the right multiplier. We have now proved that $\hat{\delta}$ is a proper action with respect to $\mathcal{D}$.

A glance at (3.7) shows that each $\langle x, y\rangle$ belongs to $B \times_{\delta, r}(G / H)$; we need to argue that $\operatorname{sp}\{\langle x, y\rangle: x, y \in \mathcal{D}\}$ is dense in $B \times_{\delta, r}(G / H)$. Since $\delta$ is a nondegenerate coaction, $\left\{\delta_{u}(b): u \in A_{c}(G), b \in B\right\}$ is dense in $B$, and by linearity it suffices to show that each $\left((\pi \otimes \iota)\left(\delta\left(\delta_{u}(b)\right)\right)\right)(1 \otimes M \mid(\Phi(f)))$ can be approximated by some $\langle x, y\rangle$.

Since $\mathcal{D}^{2}$ is dense in $B \times{ }_{\delta} G$, there exist $x_{i}, y_{i} \in \mathcal{D}$ such that

$$
x_{i}^{*} y_{i} \rightarrow\left((\pi \otimes \iota)\left(\delta\left(\delta_{u}(b)\right)\right)\right)(1 \otimes M(f)) .
$$

By applying Lemma 3.2 to the right-hand side of (3.9), we may assume there exist $g_{1}, g_{2} \in C_{c}(G)$ such that

$$
\left(1 \otimes M\left(g_{1}\right)\right)\left(x_{i}^{*} y_{i}\right)\left(1 \otimes M\left(g_{2}\right)\right) \rightarrow(\pi \otimes \iota)\left(\delta\left(\delta_{u}(b)\right)\right)(1 \otimes M(f)) .
$$

Lemma 3.3 and the continuity property of $\Psi$ [12, Corollary 3.6] give

$$
\begin{aligned}
(\pi \otimes \iota)\left(\delta\left(\delta_{u}(b)\right)\right)(1 \otimes M \mid(\Phi(f)) & =\Psi\left((\pi \otimes \iota)\left(\delta\left(\delta_{u}(b)\right)\right)(1 \otimes M(f))\right) \\
& =\Psi\left(\left(1 \otimes M\left(g_{1}\right)\right)\left(\lim _{i} x_{i}^{*} y_{i}\right)\left(1 \otimes M\left(g_{2}\right)\right)\right) \\
& =\lim _{i} \Psi\left(\left(1 \otimes M\left(g_{1}\right)\right)\left(x_{i}^{*} y_{i}\right)\left(1 \otimes M\left(g_{2}\right)\right)\right) \\
& =\lim _{i}\left\langle x_{i}\left(1 \otimes M\left(g_{1}\right)^{*}\right), y_{i}\left(1 \otimes M\left(g_{2}\right)\right)\right\rangle .
\end{aligned}
$$

Since $x_{i}\left(1 \otimes M\left(g_{1}\right)^{*}\right)$ and $y_{i}\left(1 \otimes M\left(g_{2}\right)\right)$ belong to $\mathcal{D}$ by Lemma 3.2 this proves that the generalised fixed-point algebra is all of $B \times_{\delta, r}(G / H)$.

That $\hat{\delta}$ is also saturated with respect to $\mathcal{D}$ follows from the proof of [5, Lemma 4.1]: the proof goes through if $B \times{ }_{\delta} G$ is replaced with any dense $*$-subalgebra. The last assertion now follows directly from [16, Corollary 1.7]. 
Remark 3.4. It follows from our result that the generalised fixed-point algebra associated to Rieffel's $*$-subalgebra $A_{0}:=\left(1 \otimes M\left(C_{c}(G)\right)\right)\left(B \times_{\delta} G\right)\left(1 \otimes M\left(C_{c}(G)\right)\right)$ coincides with $B \times_{\delta, r}(G / H)$. To see this, let $a, b \in A_{0}$. The calculation (3.8) shows that Rieffel's inner product $\langle a, b\rangle_{D}$ coincides with $\Psi\left(a^{*} b\right)$. Now choose $f, g \in C_{c}(G)$ such that $a^{*} b=(1 \otimes M(f)) a^{*} b(1 \otimes M(g))$, and sequences $\left\{x_{i}\right\},\left\{y_{i}\right\}$ in $\mathcal{D}$ such that $x_{i} \rightarrow a$ and $y_{i} \rightarrow b$. The continuity property of $\Psi$ described in [12, Corollary 3.6] gives

$$
\begin{aligned}
\Psi\left(a^{*} b\right) & =\Psi\left(\lim _{i}(1 \otimes M(f)) x_{i}^{*} y_{i}(1 \otimes M(g))\right) \\
& =\lim _{i} \Psi\left((1 \otimes M(f)) x_{i}^{*} y_{i}(1 \otimes M(g))\right) \\
& =\lim _{i}\left\langle x_{i}(1 \otimes M(f)), y_{i}(1 \otimes M(g))\right\rangle .
\end{aligned}
$$

So $\Psi\left(a^{*} b\right)$ is the limit of elements in $B \times_{\delta, r}(G / H)$ and is itself in $B \times_{\delta, r}(G / H)$. Since $\{\langle x, y\rangle: x, y \in \mathcal{D}\}$ spans a dense subspace of $B \times_{\delta, r}(G / H)$, so does $\left\{\langle a, b\rangle_{D}\right.$ : $\left.a, b \in A_{0}\right\}$.

If $\nu$ is a nondegenerate representation of $B \times{ }_{\delta, r}(G / H)$ on $\mathcal{H}$, we can induce it to a representation $\overline{\mathcal{D}}$-Ind $\nu$ of $\left(B \times_{\delta} G\right) \times_{\hat{\delta}, r} H$ on $\overline{\mathcal{D}} \otimes_{B \times_{\delta, r}(G / H)} \mathcal{H}$. Composing $\overline{\mathcal{D}}$-Ind $\nu$ with the canonical map of $B \times_{\delta} G$ into $M\left(\left(B \times_{\delta} G\right) \times_{\hat{\delta}} H\right)$ gives a representation $\operatorname{Ind}_{G / H}^{G} \nu$ of $B \times{ }_{\delta} G$ which we can reasonably call the representation of $B \times{ }_{\delta} G$ induced from $\nu$. There is also a unitary representation $U$ of $H$ such that $\left(\operatorname{Ind}_{G / H}^{G} \nu, U\right)$ is a covariant representation of $\left(B \times_{\delta} G, H, \hat{\delta}\right)$, and the representation $\left(\operatorname{Ind}_{G / H}^{G} \nu\right) \times U$ of $\left(B \times{ }_{\delta} G\right) \times{ }_{\hat{\delta}} H$ factors through the representation $\overline{\mathcal{D}}$-Ind $\nu$ of the reduced crossed product. If $H$ is amenable, then $\left(B \times{ }_{\delta} G\right) \times{ }_{\hat{\delta}} H=\left(B \times{ }_{\delta} G\right) \times{ }_{\hat{\delta}, r} H$, and it follows from the Rieffel correspondence that every representation $\mu \times U$ of $\left(B \times{ }_{\delta} G\right) \times{ }_{\hat{\delta}} H$ is equivalent to one of the form $\overline{\mathcal{D}}$-Ind $\nu$. Thus we have the following imprimitivity theorem:

Corollary 3.5. Suppose $\delta$ is a nondegenerate reduced coaction of a locally compact group $G$ on a $C^{*}$-algebra $B, H$ is a closed amenable subgroup of $G$, and $\mu$ is a nondegenerate representation of $B \times{ }_{\delta} G$ on $\mathcal{H}$. Then $\mu$ is unitarily equivalent to a representation induced from a representation $\nu$ of $B \times_{\delta, r}(G / H)$ if and only if there is a unitary representation $U$ of $H$ on $\mathcal{H}$ such that $(\mu, U)$ is a covariant representation of $\left(B \times{ }_{\delta} G, H, \hat{\delta}\right)$.

\section{Normal Subgroups}

Throughout this section, $N$ is a closed normal subgroup of $G$, so that $G / N$ is itself a locally compact group. We will show how to restrict the coaction $\delta$ to a coaction $\delta \mid$ of $G / N$, and that the crossed product by $\delta \mid$ is then isomorphic to $B \times{ }_{\delta, r}(G / N)$. We therefore obtain an imprimitivity theorem relating $\left(B \times{ }_{\delta} G\right) \times_{\hat{\delta}, r} N$ to $B \times \times_{\delta \mid}(G / N)$. From this we can deduce a theorem of Kaliszewski and Quigg [6] Theorem 3.3] which extends Mansfield imprimitivity to full coactions and normal nonamenable subgroups. Our main innovation here is the concept of restriction for reduced coactions, which is based on work of Quigg [13].

Let $\delta: B \rightarrow M\left(B \otimes C_{r}^{*}(G)\right)$ be a nondegenerate coaction. The quotient map $q$ : $G \rightarrow G / N$ induces a homomorphism $q: C^{*}(G) \rightarrow C^{*}(G / N)$, and the composition $\lambda^{G / N} \circ q$ is the induced representation $\operatorname{Ind}_{N}^{G}\left(1^{N}\right)$. If $N$ is amenable, then $\operatorname{ker} 1^{N} \supset$ 
$\operatorname{ker} \lambda^{N}$. So

$$
\operatorname{ker}\left(\lambda^{G / N} \circ q\right)=\operatorname{ker} \operatorname{Ind}_{N}^{G}\left(1^{N}\right) \supset \operatorname{ker} \operatorname{Ind}\left(\lambda^{N}\right)=\operatorname{ker} \lambda^{G},
$$

and $q$ descends to a homomorphism $q^{r}: C_{r}^{*}(G) \rightarrow C_{r}^{*}(G / N)$. For amenable subgroups $N$, the restriction is usually defined by $\delta \mid:=\left(\iota \otimes q^{r}\right) \circ \delta$. For this definition to work, however, we do not need that $\lambda^{G / N} \circ q$ factors through $C_{r}^{*}(G)$, only that $\iota \otimes\left(\lambda^{G / N} \circ q\right)$ factors through $B \otimes C_{r}^{*}(G)$, and this happens more frequently than one might expect: for example, it follows from the absorbing property of the regular representation that this happens for $B=C_{r}^{*}(G)$. So there remains the possibility of defining restrictions even when $N$ is not amenable. The usual way to get around this problem of defining restrictions is to consider only full coactions $\epsilon: B \rightarrow M\left(B \otimes C^{*}(G)\right)$, because then $\epsilon \mid:=(\iota \otimes q) \circ \epsilon: B \rightarrow M\left(B \otimes C^{*}(G / N)\right)$ always makes sense.

A full coaction is Quigg-normal if the canonical map $j_{B}$ of $B$ into $M\left(B \times_{\epsilon} G\right)$ is injective [13, Definition 2.1]. Quigg showed in [13, Theorem 4.7] that every nondegenerate reduced coaction $\delta$ of $G$ on $B$ admits a unique Quiggification: a nondegenerate full Quigg-normal coaction $\delta^{Q}: B \rightarrow M\left(B \otimes C^{*}(G)\right)$ with reduction $\left(\delta^{Q}\right)^{r}:=\left(\iota \otimes \lambda^{G}\right) \circ \delta^{Q}$ equal to $\delta$. If $N$ is amenable, the equation $\lambda^{G / N} \circ q=q^{r} \circ \lambda^{G}$ implies that $\delta\left|=\left(\delta^{Q}\right)^{r}\right|=\left(\delta^{Q} \mid\right)^{r}$. Thus we feel free to define:

Definition 4.1. Let $\delta: B \rightarrow M\left(B \otimes C_{r}^{*}(G)\right)$ be a coaction of $G$ on $B$. Then the restriction of $\delta$ to a coaction of $G / N$ is $\delta \mid:=\left(\delta^{Q} \mid\right)^{r}: B \rightarrow M\left(B \otimes C_{r}^{*}(G / N)\right)$.

The crucial points about this definition are, first, that it makes sense for any normal subgroup, amenable or not, and, second, that the crossed product by $\delta$ coincides with $B \times_{\delta, r}(G / N)$. To see this second point, we consider the canonical covariant representation $\left(j_{B}, j_{G}\right)$ of $\left(B, G, \delta^{Q}\right)$ in $M\left(B \times{ }_{\delta^{Q}} G\right)$, and the restriction $j_{G} \mid$ of $j_{G}$ to $C_{0}(G / N) \subset M\left(C_{0}(G)\right)$. Then $\left(j_{B}, j_{G} \mid\right)$ is a covariant representation of $\left(B, G / N, \delta^{Q} \mid\right)$ in $M\left(B \times \delta_{\delta^{Q}} G\right)$, and because $\delta^{Q}$ is Quigg-normal, 6] Lemma 3.2] implies that $j_{B} \times j_{G} \mid$ is an injective homomorphism of $B \times{ }_{\delta^{Q} \mid}(G / N)$ into $M\left(B \times{ }_{\delta^{Q}}\right.$ $G)$; its range is spanned by elements of the form $j_{B}(b) j_{G} \mid(f)$ for $f \in C_{0}(G / N)$. Since $B \times{ }_{\delta^{Q}} G$ is canonically isomorphic to $B \times{ }_{\left(\delta^{Q}\right)^{r}} G=B \times{ }_{\delta} G$ [15, Theorem 4.1], and this isomorphism carries $\overline{\operatorname{sp}}\left\{j_{B}(b) j_{G} \mid(f): f \in C_{0}(G / N)\right\}$ onto $B \times \times_{\delta, r}(G / N)$, we have:

Proposition 4.2. Let $\delta$ be a nondegenerate reduced coaction of $G$ on $B$, let $N$ be a closed normal subgroup of $G$, and let $\delta \mid$ be the restriction to a reduced coaction of $G / N$ as in Definition 4.1. Then $B \times{ }_{\delta \mid}(G / N)$ is isomorphic to the crossed product $B \times_{\delta, r}(G / N)$ of $B$ by the homogeneous space $G / N$.

Corollary 4.3. Let $\delta$ be a nondegenerate reduced coaction of $G$ on $B$. Then Mansfield's subalgebra $\mathcal{D}$ of $B \times{ }_{\delta} G$ completes to give a Morita equivalence between $\left(B \times{ }_{\delta} G\right) \times_{\hat{\delta}, r} N$ and $B \times \times_{\delta \mid}(G / N)$.

Using the trick of passing from full to reduced crossed products we obtain a new proof of the Kaliszewski-Quigg version of Mansfield's imprimitivity theorem using Corollary 4.3.

Corollary 4.4 ([6, Corollary 3.4]). Suppose $\epsilon: B \rightarrow M\left(B \otimes C^{*}(G)\right)$ is a nondegenerate full coaction and $N$ is a closed normal subgroup of $G$. If the natural homomorphism $j_{B} \times j_{G} \mid: B \times_{\epsilon \mid}(G / N) \rightarrow B \times{ }_{\epsilon} G$ is injective, then $\mathcal{D}$ completes to give a Morita equivalence between $\left(B \times{ }_{\epsilon} G\right) \times_{\hat{\epsilon}, r} N$ and $B \times{ }_{\epsilon \mid}(G / N)$. 
Proof. Let $I=\operatorname{ker}((\iota \otimes \lambda) \circ \epsilon)$, and consider the reduction $\epsilon^{r}: B / I \rightarrow M((B / I) \otimes$ $\left.C_{r}^{*}(G)\right)$. It follows from Corollary 4.3 that $\mathcal{D}$ completes to give a Morita equivalence between $\left((B / I) \times \times_{\epsilon^{r}} G\right) \times \times_{\widehat{\epsilon}^{r}, r} N$ and $(B / I) \times_{\epsilon^{r} \mid}(G / N)$. By [15, Theorem 4.1] there is an isomorphism $\Omega$ of $(B / I) \times{ }_{\epsilon^{r}} G$ onto $B \times{ }_{\epsilon} G$ such that

$$
\Omega\left(j_{B / I}^{r}(q(b)) j_{G}(f)\right)=j_{B}(b) j_{G}(f) .
$$

Thus $\Omega$ preserves the dual actions, and induces an isomorphism of $\left((B / I) \times{ }_{\epsilon^{r}}\right.$ $G) \times \times_{\widehat{\epsilon}^{r}, r} N$ onto $\left(B \times{ }_{\epsilon} G\right) \times \times_{\hat{\epsilon}, r} N$. On the other hand, $\Omega$ also extends to an isomorphism $\bar{\Omega}$ of $M\left((B / I) \times{ }_{\epsilon^{r}} G\right)$ onto $M\left(B \times{ }_{\epsilon} G\right)$ such that

$$
\bar{\Omega}\left(j_{B / I}^{r}(q(b)) j_{G} \mid(f)\right)=j_{B}(b) j_{G} \mid(f),
$$

which implies that $\bar{\Omega}$ restricts to an isomorphism of $(B / I) \times_{\epsilon^{r}, r}(G / N)$ onto the range of $j_{B} \times j_{G} \mid$. Now it follows from Proposition 4.2 and the injectivity of $j_{B} \times j_{G} \mid$ that we have a natural isomorphism of $(B / I) \times_{\epsilon^{r} \mid}(G / N)$ onto $B \times{ }_{\epsilon \mid}(G / N)$, and the result follows.

Remark 4.5. Because we have made sense of restriction for reduced coactions, we can also deduce Corollary 4.3 from the results of [6] by applying [6, Corollary 3.4] to the Quiggification $\delta^{Q}$, which is always Quigg-normal.

\section{REFERENCES}

[1] K. Deicke, D. Pask, and I. Raeburn, Coverings of directed graphs and crossed products of $C^{*}$-algebras by coactions of homogeneous spaces, Internat. J. Math., to appear.

[2] S. Echterhoff, S. Kaliszewski, and I. Raeburn, Crossed products by dual coactions of groups and homogeneous spaces, J. Operator Theory 39 (1998), 151-176. MR 99h:46124

[3] S. Echterhoff and J. Quigg, Full duality for coactions of discrete groups, Math. Scand. 90 (2002), 267-288. MR 2003g:46079

[4] P. Green, The local structure of twisted covariance algebras, Acta Math. 140 (1978), 191-250. MR 58:12376

[5] A. an Huef, I. Raeburn, and D. P. Williams, A symmetric imprimitivity theorem for commuting proper actions, preprint (arXiv.math.OA/0202046), 2002.

[6] S. Kaliszewski and J. Quigg, Imprimitivity for $C^{*}$-coactions of non-amenable groups, Math. Proc. Cambridge Philos. Soc. 123 (1998), 101-118. MR 99a:46118

[7] M. B. Landstad, J. Phillips, I. Raeburn, and C. E. Sutherland, Representations of crossed products by coactions and principal bundles, Trans. Amer. Math. Soc. 299 (1987), 747-784. MR 88f:46127

[8] K. Mansfield, Induced representations of crossed products by coactions, J. Funct. Anal. 97 (1991), 112-161. MR 92h:46095

[9] C. K. Ng, A remark on Mansfield's imprimitivity theorem, Proc. Amer. Math. Soc. 126 (1998), 3767-3768. MR 99h:46111

[10] D. Olesen and G. K. Pedersen, Applications of the Connes spectrum to $C^{*}$-dynamical systems, J. Funct. Anal. 30 (1978), 179-197. MR 81i:46076a

[11] D. Olesen and G. K. Pedersen, Applications of the Connes spectrum to $C^{*}$-dynamical systems II, J. Funct. Anal. 36 (1980), 18-32. MR 81i:46076b

[12] J. C. Quigg, Landstad duality for $C^{*}$-coactions, Math. Scand. 71 (1992), 277-294. MR 94e: 46119

[13] J. C. Quigg, Full and reduced $C^{*}$-coactions, Math. Proc. Cambridge Philos. Soc. 116 (1994), 435-450. MR 95g:46126

[14] J. C. Quigg and I. Raeburn, Induced $C^{*}$-algebras and Landstad duality for twisted coactions, Trans. Amer. Math. Soc. 347 (1995), 2885-2915. MR 95j:46080

[15] I. Raeburn, On crossed products by coactions and their representation theory, Proc. London Math. Soc. 64 (1992), 625-652. MR 93e:46080

[16] M. A. Rieffel, Proper actions of groups on $C^{*}$-algebras, Mappings of Operator Algebras, Progr. Math., vol. 84, Birkhäuser, Boston, 1988, pp. 141-182. MR 92i:46079 
[17] M. A. Rieffel, Integrable and proper actions on $C^{*}$-algebras, and square integrable representations of groups, preprint (arXiv.math.OA/9809098), 1999.

School of Mathematics, The University of New South Wales, Sydney, NSW 2052, Australia

E-mail address: astrid@maths.unsw.edu.au

School of Mathematical and Physical Sciences, University of Newcastle, NSW 2308, Australia

E-mail address: iain@frey.newcastle.edu.au 